\title{
Prevalence and potential determinants of obesity in Northern Irish schoolchildren, using geographical information systems (GIS)
}

\author{
L. King ${ }^{1}$, L. O’Donoghue ${ }^{1}$, K. J. Saunders ${ }^{1}$, J. M. McClelland ${ }^{1}$, A. J. Moore ${ }^{2}$, A. R. Rudnicka ${ }^{3}$, \\ C. G. Owen ${ }^{3}$ and A. M. Gallagher ${ }^{1}$ \\ ${ }^{1}$ School of Biomedical Sciences and ${ }^{2}$ School of Environmental Sciences, University of Ulster, Coleraine BT52 1SA, UK \\ and ${ }^{3}$ Community Health Services, St George's, University of London, London SW17 ORE, UK
}

The increased prevalence of childhood obesity is linked with several behavioural and social factors ${ }^{(1)}$. Socio-economic deprivation has traditionally been associated with poorer health and increased prevalence of overweight-obesity ${ }^{(2)}$. The aim of the present study was to examine behavioural and social correlates of childhood overweight-obesity in a group of Northern Irish schoolchildren using data collected as part of a cluster-sampled cross-sectional study of schoolchildren. Data collected included anthropometry (height, weight) and child and/or parental questionnaires on early-life exposures (including birth weight and early infant feeding practices), physical activity (self-reported) and parental adiposity (self-reported). A GIS approach, using unit postcode address information and the Northern Ireland multiple deprivation measure (NIMDM 2005), was applied to assign an area-based rank measure of economic deprivation to each child ${ }^{(3)}$. The measure, calculated at the small-scale census output area (OA) level, is based on three weighted domains of deprivation: income $(41.7 \%)$; employment $(41.7 \%)$; proximity to services (16.6\%). Associations were estimated using logistic regression analysis and expressed as unadjusted or adjusted (for gender and/or parental weight status) OR and $95 \%$ CI.

In total 1066 children (398 aged 6-7 years, 668 aged 12-13 years) participated, with similar numbers of boys and girls. The prevalence of overweight-obesity using UK90 definitions ${ }^{(4)}$ was $20.8 \%$ in the 6-7 year olds and $33.3 \%$ in the $12-13$ year olds. Unadjusted OR showed that higher birth weight $(>3.5 \mathrm{~kg})$ was associated with a 2.4-fold increased risk of being overweight-obese at age 6-7 years $(P=0.004)$ whereas in the 12-13-year-old group female gender and low physical activity levels was associated with an increased risk of being overweight-obese $(1.7$-fold and 2.3 -fold respectively, both $P=0.001)$. In both age-groups unadjusted OR showed that having two parents who were overweight or obese was the strongest predictor of being overweight-obese in childhood (for the 6-7 year olds OR 3.5 (95\% CI 1.6, 7.8), $P=0.002$; for the 12-13 year olds OR 2.7 (95\% CI 1.4, 5.0), $P=0.003$ ). In the final models (adjusted for gender and/or parental adiposity status) these associations held with the factors most significantly associated with childhood obesity being birth weight $>3.5 \mathrm{~kg}$ in 6-7 year olds and low physical activity and female gender in the 12-13 year olds. In both age-groups childhood obesity was strongly associated with both parents being overweight-obese (for 6-7 year olds OR 3.7 (95\% CI 1.7, 8.5); 12-13 year olds OR 2.7 (95\% CI 1.4, 5.2); both $P<0.001$ ). In contrast to other methods used for categorising socio-economic deprivation at the individual level, the GIS approach represented a low subject burden method and avoided the need for intrusive questioning about personal circumstances. Outcomes from GIS revealed no significant difference between small-scale area deprivation and risk of overweight and obesity in children. Family-based strategies to combat parental obesity and encourage physical activity in younger Northern Irish individuals are needed urgently.

1. Government Offices for Science (2007) Foresight. Tackling obesities: future choices - project report. http://www.foresight.gov.uk/Obesity/17.pdf (accessed March 2009).

2. Wang Y (2001) Int J Epidemiol 30, 1129-1136.

3. Northern Ireland Statistic and Research Agency (2005) Northern Ireland Multiple Deprivation Measures. http://www.nisra.gov.uk/archive/deprivation/ NIMDM2005FullReport.pdf (last accessed March 2009).

4. Cole TJ, Freemann JV \& Preece MA (1995) Arch Dis Child 73, 25-29. 